\title{
Vulnerabilidad Territorial y Accesibilidad a los Servicios de Proximidad para las Personas Mayores en la Ciudad de Valencia*
}

\section{Territorial Vulnerability and Accessibility to Proximity Services for Older People in the City of Valencia}

\author{
María Dolores Pitarch-Garrido \\ maria.pitarch@uv.es @ https://orcid.org/0000-0001-9109-4687 \\ Félix Fajardo-Magraner \\ felix.fajardo@uv.es @ https://orcid.org/0000-0003-1516-6655
}

Departamento de Geografía. Instituto Interuniversitario de Desarrollo Local. Universitat de València

Av. Blasco Ibañez, 28. 46010 Valencia

\section{INFO ARTÍCULO}

Enviado: $21 / 3 / 2019$

Revisado: 21/5/2019

Aceptado: 22/5/2019

\section{PALABRAS CLAVE}

Servicios públicos

Movilidad sostenible

Accesibilidad

Pobreza senior

Población mayor

Valencia

\section{KEYWORDS}

Public services

Sustainable mobility

Accessibility

Senior poverty

Elderly population

Valencia

\section{RESUMEN}

El aumento de la edad media de la población en las sociedades occidentales es una realidad que define nuevos parámetros de convivencia a la vez que nuevas demandas sociales. En el caso de los mayores, los servicios garantizan no sólo el cuidado, en particular a los dependientes, sino también la compañía (soledad). En este artículo se analiza la localización y accesibilidad a dichos servicios, concretamente centros de día, centros municipales de actividad y residencias, en la ciudad de Valencia, lo cual resulta de gran interés también para la planificación y gestión pública. Se hace hincapié en el contexto de los barrios más vulnerables. Los resultados nos indican que existen claras diferencias según el lugar de residencia de las personas, el modelo público o privado de la oferta y el tipo de transporte, todo lo cual resta cohesión social y espacial, a pesar de la buena situación general de la ciudad.

* Este artículo se ha elaborado en el marco del proyecto "Sostenibilidad social, conectividad global y economía creativa como estrategias de desarrollo en el Área metropolitana de Valencia" (CSO2016-74888-C4-1-R), financiado por la Agencia Estatal de Investigación (AEI) y al Fondo Europeo de Desarrollo Regional (FEDER) dentro del Programa Estatal de Investigación, Desarrollo e Innovación Orientada a los Retos de la Sociedad, en el marco del Plan Estatal de Investigación Científica y Técnica y de Innovación 2013-2016, convocatoria de 2016.Uno de los autores, Félix Fajardo, cuenta con una ayuda para contratos predoctorales para la formación de profesorado universitario, convocatoria 2014 (FPU14/05550), financiada por el Ministerio de Educación, Cultura y Deporte. 


\section{INTRODUCCIÓN}

Una de las características indiscutibles de la sociedad occidental actual es la elevada edad media de la población, originada por una bajísima tasa de natalidad y una mayor esperanza de vida. Esta situación, que ya tiene poco de coyuntural, determina algunas de las actuaciones públicas (sobre todo en lo que tiene que ver con la fiscalidad y las pensiones) y muchas de las acciones privadas, en particular en aquellos países o regiones donde la familia sigue siendo el sustento fundamental de la sociedad.

Según la Organización Mundial de la Salud (OMS) (2012)' el envejecimiento de la población es más rápido que nunca antes en la historia. Entre 2015 y 2050 el aumento de la población mayor de 60 años a nivel mundial pasará del 12 al 22\%, rebasando los 2.000 millones de personas, y no hay pruebas que indiquen que las personas mayores gocen ahora de mejor salud que sus padres, es decir que las limitaciones vitales son prácticamente las mismas. Además, según este mismo estudio, se empieza a detectar un aumento de la discriminación por razones de edad, incluso superior a la discriminación por sexo o raza, en forma de prejuicios, actitudes y prácticas o políticas, lo que puede suponer, según la OMS, una desvalorización y menoscabo de la calidad de la atención sanitaria y social a las personas mayores.

A nivel local, en concreto a escala urbana, el envejecimiento de la población supone un reto importantísimo para cuestiones prácticas básicas, tanto a nivel público como privado: desde el transporte hasta los servicios a domicilio, y, por supuesto, los cuidados, cuando son necesarios. La calidad de vida de las personas no debe disminuir cuando éstas aumentan en edad $y$, aunque las situaciones personales son muy diferentes, sus derechos siguen siendo los mismos.

Como indica la OMS (2012), las personas mayores, cuyo número y proporción en los entornos urbanos aumentará de forma considerable, presentan unos elevados índices de exclusión, problema que se une a otros como la soledad y la mala salud, la dificultad (por precio o por lejanía) para acceder a los mecanismos de protección social, entre ellos, los servicios públicos básicos. Son problemas que las políticas de austeridad en tiempos de crisis no han hecho sino acrecentar (OCDE, 2009, Doyle et al., 2009, Falkingham et al., 2012, OMS, 2012, OMS, 2015, Abellán y Pujol, 2016, Nieto-Masot et al., 2019).

El siglo XXI ha visto aparecer el término "envejecimiento activo". Para la OMS (2001) el envejecimiento activo es "el proceso de aprovechar al máximo las oportunidades para tener un bienestar físico, psíquico y social durante toda la vida. El objetivo es extender la calidad y esperanza de vida a edades avanzadas. Además de continuar siendo activo físicamente, es importante permanecer activo social y mentalmente participando en: actividades recreativas, actividades con carácter voluntario o remuneradas, actividades culturales y sociales, actividades educativas, vida diaria en familia y en la comunidad." (p.2) y añade: "El envejecimiento activo es positivo para todos, sin consideración alguna de las facultades psíquicas o físicas ni del estado socioeconómico o ubicación geográfica de la persona." (p.2). En este sentido, resulta relevante que las condiciones materiales faciliten y, en cierta medida, garanticen el envejecimiento activo. Una parte significativa es la que tiene que ver con la autonomía de las personas mayores. Dicha autonomía puede conseguirse mediante la modificación del entorno directo que habita, el hogar, pero también mediante la generación de mecanismos de apoyo que contribuyan a satisfacer las necesidades básicas (OMS, 2015).

En resumen, en una sociedad envejecida la calidad de vida de los mayores, tanto desde el punto de vista físico como psicológico, es un valor a preservar y mejorar no sólo porque involucra a un número cada vez mayor de personas, sino porque en un Estado de Bienestar es objetivo y responsabilidad de la política pública. Así pues, un envejecimiento activo, como se ha señalado, supone protección contra la pobreza, el aislamiento y la enfermedad. En las ciudades, la oferta de servicios y la protección social presentan un determinado patrón espacial que puede marcar diferencias entre las personas según el barrio en el que residan.

Con este convencimiento, acometemos esta investigación cuyo objetivo es identificar las áreas vulnerables en la ciudad de Valencia en términos de capacidad socioeconómica de sus residentes y de la accesibilidad a la oferta de servicios a la tercera edad. Nuestra hipótesis es que, a pesar de los esfuerzos de inversión

1. OMS: https://www.who.int/topics/ageing/es/ 
y de mejora de la red de servicios, todavía existen diferencias de acceso a los mismos según el lugar de residencia, siendo las áreas centrales de la ciudad las mejor servidas, tanto desde el punto de vista de la oferta pública como privada. Para ello se aplica una metodología ya contrastada (Pitarch-Garrido, Salom y Fajardo, 2018). La finalidad del análisis es conocer, con la información disponible, qué zonas de la ciudad son más vulnerables para las personas mayores que allí residen y facilitar la identificación de los principales problemas sobre los que debe incidir la planificación pública (oferta, tipología, transporte, etc.). Todo ello se detallará en los apartados que siguen a continuación.

\section{ESTADO DEL ARTE}

\subsection{Marco teórico}

Como ya se ha señalado, el proceso de envejecimiento afecta a todas las sociedades actuales, sin embargo, éste es un fenómeno espacialmente diferenciado. En las ciudades de más de 100.000 habitantes, la media de edad de la población urbana difiere 21 años en EE.UU., 12 años en China y 10 años en España (OCDE, 2015a). Existen grandes diferencias en cuanto a la edad media de la población en el interior de las ciudades, por ejemplo, en los países del norte de Europa los centros de las ciudades albergan más población joven que la periferia, mientras que, en los países del sur y este europeo, la población mayor es más abundante en el centro que en la corona metropolitana (OCDE, 2012).

Por otra parte, es necesario hablar del fenómeno del sobreenvejecimiento, es decir, del aumento del número de personas en las edades más avanzadas. Campelo et al. (2014) utilizan el índice de envejecimiento y sobreenvejecimiento como parte del proceso de vulnerabilización en las ciudades, entendido como "aquellas transformaciones en la estructura sociodemográfica, socioeconómica o sociocultural de una ciudad o área urbana que, al modificar situaciones o tendencias características de momentos temporales anteriores, afectan a las prácticas sociales e institucionales existentes..." (p. 121). En el mundo², la población mayor de 65 años (envejecimiento) supone en la actualidad el 9\% del total, y los mayores de 80 (sobreenvejecimiento) el 1,7\%. La previsión para el 2050 es que sean el 15,8 y el 4,3\% respectivamente. En los países más desarrollados se prevé que los mayores de 80 años supongan en 2050 el 10\% de la población. En España, la previsión de la ONU para 2050 es que el 14,1\% de la población sea mayor de 80 años y el 7,9\% mayor de 85 . Las cifras son relevantes por sí mismas y evidencian la necesidad de planificar para transformar esta situación en una oportunidad y no en un problema. Un elevado número de estas personas residen en las ciudades, aunque el índice de envejecimiento y de sobreenvejecimiento en las mismas sea menor que en el entorno rural ${ }^{3}$ (Nieto-Masot et al., 2019).

La OCDE (2015b) realiza una serie de recomendaciones estratégicas para la acción política en las ciudades, de cara a su desarrollo sostenible y equitativo, y dos de ellas están directamente relacionadas con el envejecimiento de la población: la ordenación de las infraestructuras y servicios urbanos para responder a las necesidades de la población mayor, y la promoción de la inclusión social y la participación de la población mayor en el diseño y gestión del entorno urbano.

Según Iglesias Souto et al. (2018), las instituciones públicas deben asumir mayor responsabilidad en la atención a las personas mayores, facilitando servicios que respondan a sus necesidades. Estos autores identifican, a partir de publicaciones científicas ${ }^{4}$, las variables predictoras del uso de los servicios a la tercera

2. ONU: https://population.un.org/wpp/Download/Standard/Population/

3. Siguiendo a Sancho Comins y Moreno Reinoso (2012), la delimitación de rural según EUROSTAT "utiliza dos criterios para cuantificar la población residente en zonas urbanas: uno relativo a la densidad y otro al tamaño demográfico mínimo. En primer lugar, las «celdas» del territorio de 1 kilómetro cuadrado han de tener una densidad de población superior a 300 hab/km2 para ser consideradas urbanas; en segundo lugar, el conjunto de celdas adyacentes que superen ese umbral de densidad han de tener una población mínima de 5.000 habitantes." (p. 614).

4. Los autores realizan una revisión sistemática a través de Psyinfo, Web of Science, Dialnet, CSIC y Teseo, seleccionando 44 artículos científicos utilizando como estrategia de búsqueda palabras clave relacionadas con personas mayores, cuidados, provisión de servicios, etc. Todos los artículos seleccionados tratan sobre el perfil o los factores que predicen el uso de los servicios sociales para mayores. 
edad. Dichas variables son: la dependencia funcional, el deterioro cognitivo, la ausencia o escasez de apoyo social y la precariedad de la vivienda. Estas dos últimas, ligadas a los temas de soledad y calidad material del barrio, justifican, en cierta medida, la necesidad de una distribución equilibrada de los servicios, al menos los de carácter público (Roberts y Chapman, 2017).

La soledad en la vejez es un problema cuando ésta no es deseada. La escasez o falta de relaciones sociales en el caso de las personas mayores tiene consecuencias en la salud física y psicológica (Yanguas et al. 2018). A ello añaden Silva et al. (2017) que es fundamental promover la independencia y la autonomía de las personas mayores. Se les debe facilitar la información necesaria y adecuada para apoyar sus decisiones diarias y estar alerta ante situaciones de desventaja y desigualdad. En definitiva, que la edad no sea causa de pérdida de capacidad de decisión y de actuación, por una inadecuada visión social de la vejez.

Tras lo señalado anteriormente, se prevé que las ciudades de los países occidentales, en particular las europeas, serán crecientemente vulnerables, precisamente debido al aumento de la población mayor, así como de la población dependiente, y al declive poblacional. Sin embargo, en Europa se le ha prestado menos atención a este tema que en otros contextos (OCDE, Japón, Corea). McCann (2017) señala la importancia de un enfoque urbano, o regional, del declive poblacional o envejecimiento por la importancia que tiene en términos de política pública, en especial en cuanto a la capacidad a largo plazo que tienen los gobiernos locales para proveer de servicios a la población mayor.

En esta línea, y focalizando la atención en el nivel local, cabe destacar lo que Amin (2002) llama la "micropolítica del contacto y el encuentro" (p. 959), es decir, las relaciones con las personas y con el entorno más cercano, el lugar en el que se vive, se trabaja y se siente como propio. Ese entorno es fundamental para la acción política de cercanía, sobre todo en cuanto a la política social se refiere.

La estructura espacial de la oferta de los servicios públicos y privados, pero sobre todo los primeros, determina las pautas de movilidad de la población y el hecho de que ésta sea más o menos sostenible. Además, por lo que respecta a la oferta de servicios públicos, uno de los condicionantes de su eficacia y eficiencia es la elección de un buen emplazamiento, lo cual se encuentra implícitamente vinculado a una buena accesibilidad. Los servicios de proximidad, los más frecuentemente demandados por la población, suponen un determinado uso del tiempo con implicaciones en la calidad de vida. Las altas densidades de población unidas a una extensa oferta de transporte público y a un espacio urbano caracterizado por una determinada mezcla de usos, son factores que contribuyen de manera trascendente a la configuración de un espacio urbano equilibrado y menos proclive a la aparición de espacios de exclusión.

Diferentes estudios (Andreotti et al., 2012, Marquet y Miralles, 2014, Blockland et al., 2016, Pitarch-Garrido, Salom y Fajardo, 2018) señalan que la movilidad más sostenible es aquella que se realiza a pie o en bicicleta, evitando el uso de cualquier medio de transporte motorizado. Si a ello añadimos que una buena accesibilidad supone cercanía a la demanda, y que la población tiene derecho (capítulo tercero de la Constitución Española) a una serie de servicios públicos considerados como de proximidad (servicios de salud, educativos y sociales básicos, así como de la administración del Estado provistos por las corporaciones locales), resulta relevante el estudio de los mismos -y los equipamientos a ellos asociados- por sus dos características fundamentales: en primer lugar, son una vía directa para la redistribución espacial y social de las rentas públicas; $y$, en segundo lugar, como consecuencia de lo anterior, su oferta por parte de la administración pública los convierte en instrumentos de las políticas de desarrollo económico, social y territorial (Nieto-Masot et al., 2019).

\subsection{Situación en la ciudad de Valencia}

Siguiendo el argumento del apartado anterior, cabe señalar que, en 2018, en la ciudad de Valencia, el índice de envejecimiento es del 20,58\% y el de sobreenvejecimiento del 11,5\% (según definición del INE, es decir, con los mayores de 85 años como porcentaje de los mayores de 65). Este último, por comparar, en la Comunidad Valenciana asciende a 14,12\%, en España es de 15,4\% y en el mundo del 8,84\%. 
La población mayor de 64 años asciende a 164.341 personas, de los que 25.177 tienen más de 85 años. La población total de la ciudad es, en 2018, de 798.538 habitantes, por lo que, como ya hemos señalado, una quinta parte de la población es mayor de 65 años, y en crecimiento. Como indican Pinazo y Berjano (2003), la cohorte de personas mayores de 65 años ha experimentado un enorme crecimiento en la ciudad de Valencia, mientras que la población total apenas ha experimentado incremento alguno. Es una ciudad envejecida y con una importante representación de la mujer, como es habitual en las edades más avanzadas. A estos datos hay que añadir el número de personas mayores que viven solas. En este sentido, los autores anteriormente citados (Pinazo y Berjano, 2003) estiman que, en 2001, el 27,5\% de los mayores de 65 años viven solos, un total de 32.468 personas. Esta cifra alcanza el 29,5\% (43.124 personas) en 2018, según información de la Oficina de Estadística del Ayuntamiento de Valencia.

La distribución de la población mayor en la ciudad no es homogénea. Los distintos barrios presentan situaciones muy diferentes, y lo mismo ocurre con la población mayor de 65 años que vive sola. Los barrios más envejecidos (mayor índice de envejecimiento) son Mahuella, Ciutat Jardí, El Palmar, Cases de Bàrcena y Marxalenes. Son barrios de la periferia urbana, de antigua tradición agrícola y que hoy se encuentran vinculados a la ciudad, pero con escasa población residente. Sin embargo, la mayor concentración de personas de la tercera edad en números absolutos, se da en la primera periferia, es decir, en los barrios del segundo ensanche y Russafa, Orriols, Aiora, Nou Moles y Mont-Olivet, zonas de nueva construcción en los años 60, entonces ocupadas por familias jóvenes, hoy población anciana. Los barrios del primer ensanche y de Ciutat Vella (centro histórico) son los que han experimentado renovaciones urbanísticas más recientes, desde finales de los 90, experimentando dos procesos paralelos: gentrificación y reemplazo generacional, lo que supone en la actualidad una estructura de edades algo más rejuvenecida y menos hogares unifamiliares de personas mayores (mapa 1).

Los barrios que albergan un mayor número de personas mayores que viven solas son Faitanar, Els Orriols, Ciutat de les Arts, El Saler, La Carrasca, El Perellonet, El Mercat y Ciutat Universitaria. En todos ellos el porcentaje de mayores de 65 años solos sobre el total de mayores de esa edad supera el 35\%, siendo elevadísimo en el primer caso, por encima del 87\%, y especialmente relevante en el segundo, pues supone un total de 944 personas, mientras que en el resto de barrios señalados la población mayor en términos absolutos es más reducida. Como podemos observar en el mapa 2, son los barrios centrales, junto a los de la periferia oriental los que concentran más población mayor que vive sola. Se trata de barrios de antigua ocupación, tradicionales, con un parque de viviendas antiguo y una oferta de servicios, en general, consolidada y amplia.

La oferta de centros de servicios (cuadro 1) para las personas mayores tampoco se encuentra equitativamente repartida entre los distintos barrios de la ciudad. Es cierto que los Centros Municipales de Actividad, todos ellos de carácter público y gestionados por el Ayuntamiento, presentan una distribución más equilibrada. Sin embargo, las residencias, en su abrumadora mayoría privadas, presentan una mayor concentración en los barrios de la primera periferia o el ensanche, siendo inexistentes en las zonas norte y sur de la ciudad. Los centros de día presentan una distribución algo más equilibrada, siendo en su mayoría privados, todos los cuales se encuentran en la zona central, más poblada, de la ciudad.

La oferta de servicios dirigida a las personas mayores es más amplia que la presentada en el cuadro 1. Existe una serie de recursos dirigidos a las personas mayores en situación de dependencia que se ofertan en el domicilio, tales como el servicio de ayuda a domicilio y el servicio de teleasistencia. En ambos casos, en la ciudad de Valencia se oferta a través de contratos o de ONGs, como Cruz Roja. Además, existen otras posibilidades como las viviendas tuteladas (sólo una en Valencia) y la oferta de transporte adaptado, también brindadas por entidades del tercer sector social. Por otra parte, consta oferta de Centros de Atención Preventiva para Personas Mayores, centros públicos dependientes de Generalitat Valenciana, de los cuales en la Comunidad Valenciana hay 7, y en la ciudad de Valencia hay sólo 2, una oferta claramente mejorable. Por último, en la ciudad hay una Oficina de Atención al Mayor y un Defensor del mayor, este último sustentado por voluntariado.

Teniendo en cuenta que el impacto social y económico de la dependencia es creciente y, previsiblemente, se incrementará en las próximas décadas, la oferta pública de centros de día y residencias resulta estratégica. El número de personas mayores en situación de dependencia en la ciudad de Valencia en 2001 ascendía 


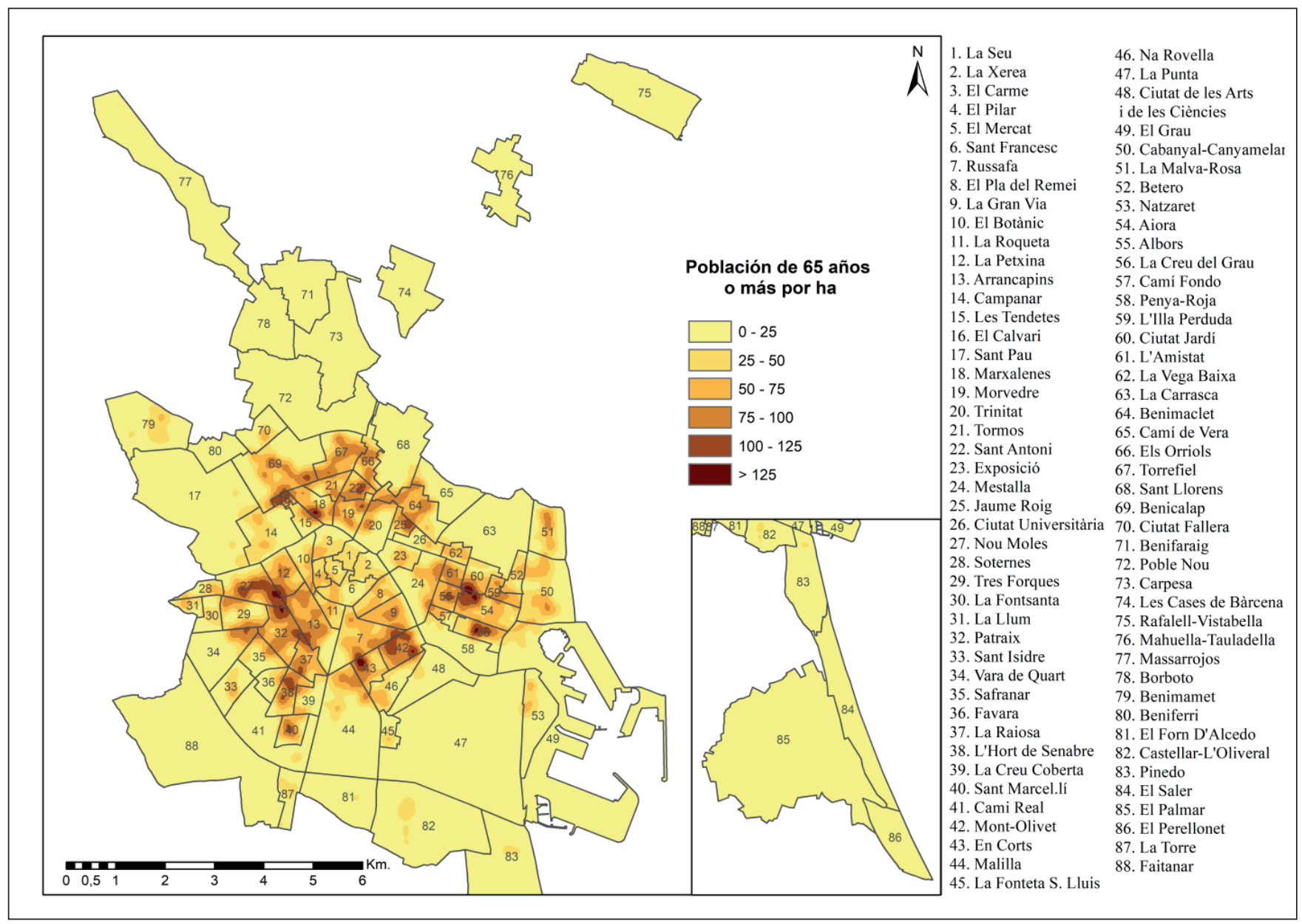

Mapa 1. Distribución de los mayores de 65 años en la ciudad de Valencia. 2018. Fuente: Elaboración propia a partir de datos de Padrón Municipal.

Oficina de Estadística del Ayuntamiento de Valencia (datos por portales).

Cuadro 1. Total de oferta de servicios (número de centros) en Valencia destinados a personas mayores.

\begin{tabular}{|l|c|c|c|}
\hline \multicolumn{1}{|c|}{ Tipo } & Total & Públicos & Privados \\
\hline Centros Municipales de Actividad & 49 & 49 & 0 \\
\hline Centros de día & 19 & 6 & 13 \\
\hline Residencias & 22 & 2 & 20 \\
\hline
\end{tabular}

Fuente: Oficina de Estadística del Ayuntamiento de Valencia.

a 23.308 (Berjano y Pinazo, 2003), mientras que, en 2018, según el Ayuntamiento de Valencia, el número de personas mayores de 65 años que tienen certificado de discapacidad es de 68.389, de las cuales, 17.643 presentan un grado de discapacidad severo, superior al 74\%. Además, en la actualidad, 2.783 personas mayores de 65 son atendidas por los servicios de Ayuda a Domicilio, 6.677 personas (de todas las edades, pero principalmente mayores) son atendidas en el Programa de Teleasistencia Domiciliaria, y 662 por el programa Menjar a Casa de los Servicios Sociales Municipales, que facilitan la comida a domicilio (Oficina Estadística del Ajuntament de València).

Las cifras expuestas confirman, por un lado, el reto que supone para la sociedad el aumento de la población mayor, y, por otro, el avance que en la última década se ha dado en la ciudad en cuanto a ofrecer respuesta a dicha demanda creciente. Cabe añadir que desde 2016 Valencia forma parte de la Red Interna- 


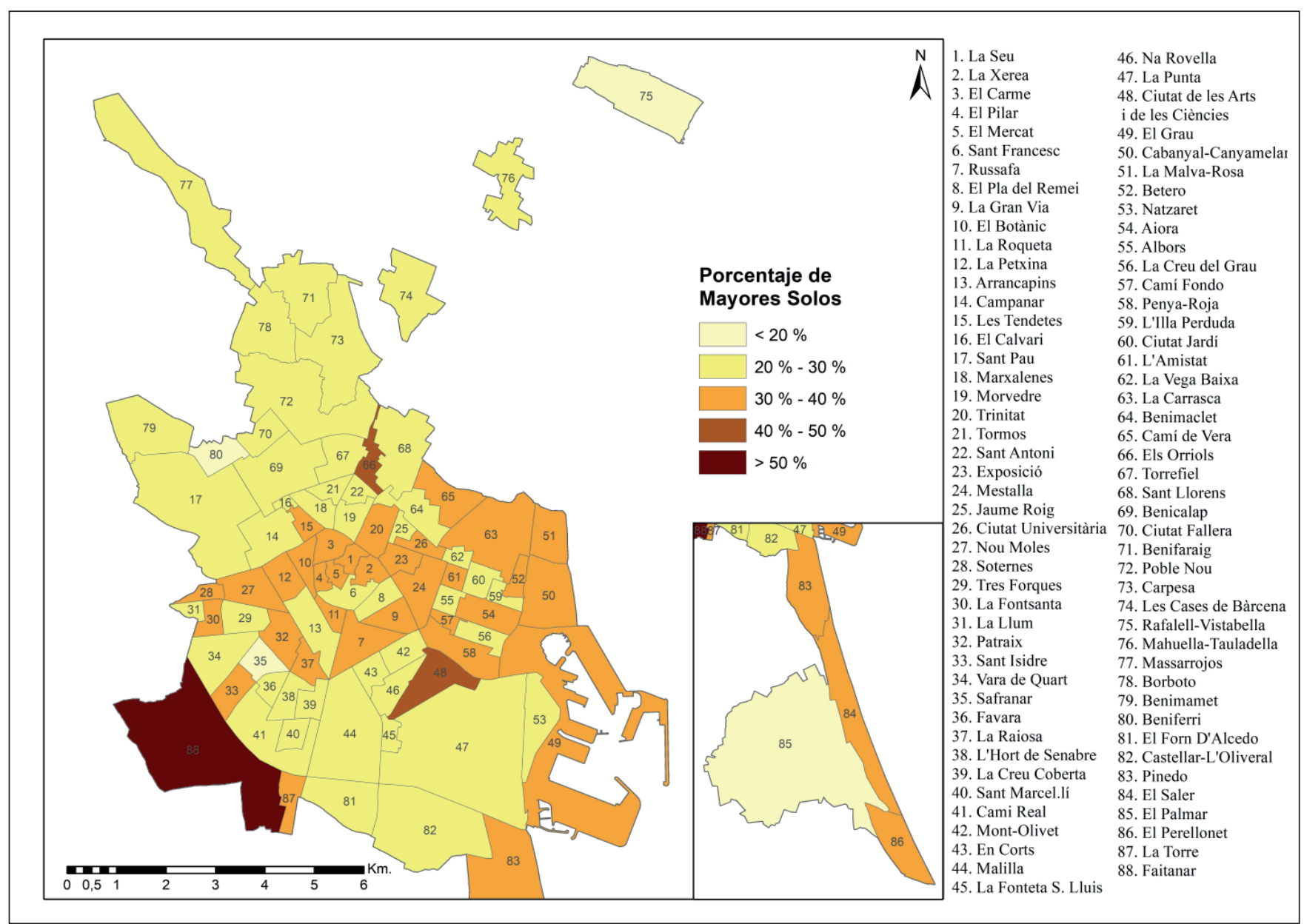

Mapa 2. Porcentaje de mayores de 65 años (sobre el total de mayores de 65) que viven solos por barrios en Valencia. 2018. Fuente: Elaboración propia a partir de datos de Oficina de Estadística del Ayuntamiento de Valencia.

cional de Ciudades Amigables con las Personas Mayores, auspiciada por la OMS, lo cual supone un primer paso, aunque sea simbólico, por hacer de la ciudad un entorno más amable para las personas mayores.

A esta situación de mejora se suma que, recientemente, en febrero de 2019, se ha aprobado la Ley 3/2019, de 18 de febrero, de servicios sociales inclusivos de la Comunitat Valenciana ${ }^{5}$, la cual supone un avance de calidad en la oferta y una reestructuración del sistema público de servicios sociales Valenciano. La puesta en marcha de esta ley supondrá una racionalización de los recursos y de la oferta, con efectos positivos para las personas mayores, pero sus efectos sólo serán detectables a largo plazo.

En nuestra investigación hemos considerado los servicios dirigidos a la población mayor con localización fija que se ofertan en la ciudad de Valencia, tanto desde el ámbito público como privado. Desde finales del siglo pasado y, sobre todo, a raíz de la crisis económico-financiera de 2008, la política que el gobierno regional y local en la Comunidad Valenciana y en su capital ha tendido hacia la restricción del gasto público, en particular en este tipo de servicios (Centros de actividades, Centros de día y Residencias), privatizando su oferta y reduciendo el número de plazas públicas. Esta política, neoliberal y de austeridad, ha supuesto recortes en las inversiones dirigidas a aumentar los recursos y equipamientos públicos para mayores.

Proponemos un nuevo vocablo para definir pobreza o exclusión que aparece, sobre todo a partir de la crisis, en este entorno urbano y social: la pobreza senior. Se trata de personas mayores, en particular muy mayores (con más de 85 años, sobreenvejecimiento), en situación de dependencia, escasa movilidad, con 
ingresos bajos (pensiones mínimas o no contributivas), que viven solas y sin entorno familiar, residentes en barrios con una baja dotación de servicios sociales públicos u obligadas a desplazarse fuera de su lugar habitual de residencia para poder acceder a servicios de cuidado. Una parte importante de esta situación de vulnerabilidad es la que nos proponemos analizar en este artículo para el caso de la ciudad de Valencia: la relacionada con el lugar, es decir, la parte que afectaría a la equidad espacial de la pobreza senior. A continuación, expondremos brevemente la metodología utilizada para pasar al análisis de la situación y las conclusiones.

\section{METODOLOGÍA}

Proponemos a continuación un estudio de caso, la ciudad de Valencia. La metodología desarrollada puede ser replicable en cualquier otro contexto urbano y ha sido ya aplicada a otro tipo de servicios públicos, por lo que no abundaremos ahora en los detalles de la misma que se pueden consultar en Pitarch-Garrido, Salom y Fajardo (2018).

Los elementos que consideramos son: la movilidad a pie (la más adecuada y sostenible para este grupo de población), la accesibilidad-cercanía, la calificación de dichos servicios como de proximidad, y, por tanto, el derecho de los usuarios a que se localice su oferta en un área dentro de su espacio vital. Los servicios considerados son: los centros municipales de actividad (conocidos como asociaciones de jubilados), los centros de día y las residencias de la tercera edad. Para el análisis se ha diferenciado entre servicios públicos, privados y el total.

En pro de la sostenibilidad de la movilidad, se ha calculado el índice de accesibilidad a los distintos centros de oferta de servicios para mayores públicos y privados a pie ${ }^{6}$ y en transporte público. Por otra parte, se analiza la cantidad de personas que suponen demanda potencial, considerando el total de la población mayor, aunque no todos sean usuarios del servicio, y se calcula el área de influencia de cada uno de los centros de oferta, comparándola con los límites de los barrios. Para cada una de las áreas resultantes, tanto las calculadas, como los barrios, se consideran las variables socioeconómicas de la población residente.

La localización de la población (según edades) en su lugar de residencia (portal o número de policía) es la base para el cálculo de la accesibilidad a los servicios. La información facilitada por el Padrón de Habitantes y cedida por la Oficina de Estadística del Ayuntamiento de Valencia ha sido de gran utilidad para la exactitud espacial de los cálculos. La medida de la accesibilidad utilizada ha sido el índice de separación espacial ${ }^{7}$ (Garrocho y Campos, 2006), en minutos. El índice se ha calculado desde cada portal al punto de oferta más cercano mediante el uso del software Arc-SIG 10.2 de ESRI.

La transformación de los datos puntuales en superficies se ha realizado a partir de la interpolación mediante el método IDW (Inverse Distance Weighting), utilizando la herramienta correspondiente del módulo Spatial Analysis de ARCINFO 10.0. El exponente de distancia utilizado ha sido de 2 aplicado a los puntos del ISE, a partir de los cuales se define un radio de búsqueda de los 12 puntos más cercanos entre sí (separados por una distancia máxima de 500 metros), y un tamaño de celda de $25 \times 25$ metros. Con estas premisas se han delimitado áreas con niveles significativos en relación con la accesibilidad.

Desde el punto de vista espacial, se ha sumado la información sociodemográfica a nivel de portal, a partir de una malla de polígonos cuadrados de 100 metros de lado dibujada sobre la ciudad de Valencia. De esta manera es posible obtener la información a una escala intermedia, entre el barrio (excesivamente amplio) y

6. La velocidad media a la que se ha considerado que se desplaza un peatón es de 4,5 km/h. Estimamos que en 10 minutos se recorren 600 metros. Las condiciones de salud de las personas mayores pueden suponer una ralentización de esta velocidad, con lo que los resultados deben ser considerados como límites de distancia máxima, es decir, para algunos mayores los 20 minutos de desplazamiento pueden convertirse en 30.

7. El Índice de Separación Espacial de la unidad espacial $i\left(I S E_{i}\right)$ es igual a: ISE $=\sum D_{i} / n$. Donde $i$ es el lugar de origen (portales) para el que se calcula el índice, $j$ es cada uno de los potenciales lugares de destino de los desplazamientos (equipamientos). Sólo se utiliza el más cercano al lugar de origen. $D_{i j}$ es la distancia en minutos, -obtenida a partir de las matrices calculadas-, entre el lugar de origen $\mathrm{i}$ y el lugar de destino j. $Y n$ es el número de posibles lugares de destino. 
el portal (difícil de representar por la multitud de puntos). A cada celda se le ha asignado el valor medio de los puntos de las residencias y el del barrio. Para la caracterización de las diversas zonas de la ciudad, junto a la información a nivel de portal (Padrón), se han considerado distintos indicadores de la situación económica del barrio en que se ubican ${ }^{8}$. Con estos datos se ha realizado un Análisis Factorial de Componentes Principales (ACP) que nos ha permitido reducir la cantidad de datos, siendo posible establecer los elementos comunes a las variables asociadas (Pitarch, Salom y Fajardo, 2018). El tratamiento estadístico se ha llevado a cabo con el programa IBM SPSS Statistics, Versión 22. Se ha utilizado el método propuesto por López-Roldán et al (2016), por tanto, se ha seleccionado el primer componente, que explica por sí mismo el 32,6\% de la varianza. El valor de este componente en sentido negativo nos da una idea muy ajustada de la localización de la población con menor nivel de renta, menor calidad funcional y del entorno, bajos precios del suelo (y de la vivienda) y escasez o ausencia de funciones superiores. Así, ha sido posible cartografiar las áreas (espacios inferiores al nivel de barrio) de la ciudad de Valencia donde se dan al mismo tiempo un nivel socioeconómico bajo con una lejanía relativa a los servicios públicos considerados.

\section{RESULTADOS Y DISCUSIÓN}

El análisis de la situación de la accesibilidad de las personas mayores a los servicios a ellas destinados en Valencia presenta algunos resultados destacables. En términos de distancias medias recorrida, medidas en tiempo, en Valencia resulta evidente que el transporte público es más competitivo (Cuadro 2). Esto no resulta siempre tan evidente, ya que la cercanía del servicio a la demanda pudiera hacerlo más accesible a pie, evitando los inconvenientes de las esperas, trasbordos, etc. del transporte público. Sin embargo, en todos los casos, es menor el tiempo medio usado por los usuarios para acceder al servicio en transporte público.

Cuadro 2. Tiempo medio en la ciudad de acceso a los diferentes servicios analizados tanto a pie como en transporte público (promedio de ISE).

\begin{tabular}{|l|l|c|c|}
\hline \multicolumn{1}{|c}{ Tipología } & \multicolumn{1}{c|}{ Titularidad } & \multicolumn{2}{c|}{ Minutos } \\
\cline { 2 - 4 } & \multicolumn{1}{|c|}{ A Pie } & En Transporte Público \\
\hline \multirow{2}{*}{ Centro Municipal de Actividad } & Total (todos públicos) & 8,09 & 5,96 \\
\hline \multirow{2}{*}{ Centro de Día } & Total (públicos y privados) & 20,60 & 10,35 \\
\cline { 2 - 4 } & Público & 29,42 & 13,54 \\
\cline { 2 - 4 } & Privado & 22,70 & 11,18 \\
\hline \multirow{2}{*}{ Residencia } & Total (públicas y privadas) & 17,79 & 9,32 \\
\cline { 2 - 4 } & Pública & 41,10 & 16,41 \\
\cline { 2 - 4 } & Privada & 17,94 & 9,45 \\
\hline
\end{tabular}

Fuente: Elaboración propia.

8. Los indicadores utilizados proceden del Padrón, del Catastro y de la Oficina de Estadísticas del Ayuntamiento de Valencia. Son los siguientes: porcentaje menores de 16 años, porcentaje 65 y más años, porcentaje de población de 24 y más años con nivel educativo inferior a Bachiller, Edad media de las viviendas, valor por $\mathrm{m}^{2} \mathrm{de}$ la vivienda, porcentaje de vehículos con menos de $80 \mathrm{CV}$ sobre el total de vehículos del barrio, porcentaje de vehículos con más de 200 CV sobre el total de vehículos del barrio, porcentaje de empresas e instituciones financieras y seguros sobre el total de empresas del barrio, porcentaje de empresas de comercio, restaurantes, hostelería y reparaciones sobre el total de empresas del barrio, porcentaje de empresas profesionales sobre el total de empresas del barrio, porcentaje ocupados en comercio, hostelería y transportes sobre el total de ocupados residentes en el barrio, porcentaje de ocupados en finanzas, derecho y seguros sobre el total de ocupados residentes en el barrio, y porcentaje de nacionalidad extranjera extracomunitario. 
El cuadro 2 nos indica la situación media en la ciudad en cuanto a accesibilidad a los centros de actividad, los centros de día y las residencias. Los datos medios ocultan realidades particulares para cada barrio, sin embargo, nos dan una visión general bastante fiable. Cabe destacar, la elevada distancia media que existe entre el lugar donde viven los ciudadanos y las residencias públicas para mayores. Llama la atención el caso de las residencias, en particular las públicas, que, al ser tan escasas -sólo dos-, en comparación con la red de residencias privadas, la accesibilidad a pie es bastante negativa, el doble de tiempo que en transporte público.

Dada la importancia de la cercanía física y la localización en el entorno habitual de vida de las personas usuarias de las residencias, estos minutos pueden suponer una barrera para el acceso, dado que, además, la oferta es mayoritariamente privada, la decisión familiar de sacar a la persona mayor de su contexto es casi una necesidad. Por lo que respecta a los centros de día públicos, la distancia media en la ciudad es de unos treinta minutos a pie y algo más de trece minutos con transporte público, lo que supone también una difícil decisión familiar que obliga, en muchas ocasiones al traslado de los usuarios en transporte privado, estando, en tal caso, la decisión menos vinculada al espacio de vida y más a la facilidad de acceso por carretera y/o disponibilidad familiar. Los centros municipales de actividades son los que presentan una mejor accesibilidad, con una media de ocho minutos a pie, apenas rebajada a seis minutos en transporte público, lo que no supone diferencia real.

Las cifras generales de la ciudad en cuanto a número de personas y de personas mayores de 65 años afectadas por la lejanía a un centro de servicios de los analizados (cuadros 3 y 4) nos dan información sobre el impacto social que tiene la localización de los mismos. Hemos considerado dos umbrales a partir de los cuales los usuarios reales y potenciales se encuentran lejos del área de cercanía al servicio que hemos establecido (este umbral puede variar): veinte minutos a pie y cuarenta en transporte público. En Valencia, 12.820 residentes se localizan a más de veinte minutos de un centro municipal de actividades para personas mayores, de ellos, 2.765 son mayores de 65 años. Tan sólo supone un 1,89\% de la población total. Si incorporamos el transporte público, sólo 24 personas mayores de 65 años se encuentran a más de cuarenta minutos de uno de estos centros. Las cifras son muy bajas, lo que indica una distribución bastante equitativa desde el punto de vista espacial.

Cuadro 3. Población en relación con la distancia a los centros municipales de actividad para personas mayores.

\begin{tabular}{|c|c|c|c|c|c|}
\hline & \multirow{3}{*}{ Población afectada } & \multicolumn{4}{|c|}{ CENTROS MUNICIPALES DE ACTIVIDAD PARA PERSONAS MAYORES } \\
\hline & & \multicolumn{2}{|c|}{ A pie (a más de 20 minutos) } & \multicolumn{2}{|c|}{$\begin{array}{c}\text { Transporte Público (a más de } 40 \\
\text { minutos) }\end{array}$} \\
\hline & & Número & $\begin{array}{c}\text { Porcentaje (\%) } \\
\text { sobre el Total } \\
\text { de población } \\
\text { Valencia }\end{array}$ & Número & $\begin{array}{c}\text { Porcentaje (\%) } \\
\text { sobre el Total de } \\
\text { población } \\
\text { Valencia }\end{array}$ \\
\hline \multirow{2}{*}{ Total Centros } & Residentes & 12.820 & 1,71 & 73 & 0,01 \\
\hline & Mayores de 65 años & 2.765 & 1,89 & 24 & 0,02 \\
\hline
\end{tabular}

Fuente: Elaboración propia.

En el caso de los Centros de día (cuadro 4), los mayores de 65 años a más de veinte minutos a pie ascienden a 26.411. Si consideramos sólo los centros públicos, el número de potenciales usuarios, es decir, de personas mayores de 65 años, a más de veinte minutos asciende a 69.560. Estos últimos suponen un 47,6\% de la población mayor de la ciudad, cifra elevada, sobre todo si consideramos que, en el caso de los centros privados, se reduce a la mitad. En este servicio, la oferta pública es claramente deficitaria.

La distancia media a las residencias para la tercera edad, como ya se ha adelantado a partir de las cifras generales, es mayor (cuadro 5). En el caso de los centros públicos, los resultados son preocupantes, pues 114.780 mayores de 65 años residentes en la ciudad de Valencia necesitan más de veinte minutos para 
trasladarse a un servicio de este tipo, aunque en el caso de las residencias privadas, la cifra se reduce a 13.912. Los barrios más perjudicados por la distancia a residencias públicas son El Perellonet, El Palmar, El Sales, Mahuella-Tauladella, Massarrojos y Pinedo, todos ellos de la periferia de la ciudad, incluidos en los denominados Pobles del Sud y Pobles del Nord. Dentro del casco urbano consolidado, destacan por su peor posición Nazaret, Malvarrosa, Cabanyal-Canyamelar, Carpesa y la Torre, a los que se unen también otros barrios periféricos como Benimamet y Cases de Bárcena. Son barrios como El Pilar, El Carme, El Mercat, El Botànic, La Seu y Sant Francesc, todos ellos en Ciutat Vella, el distrito central de la ciudad, los que se encuentran mejor servidos en el sentido de una mayor oferta y dispersión, por lo que la cercanía al usuario es más clara. Se trata, como es sabido, de barrios de alta densidad, con una población residente muy abundante y también envejecida, lo que hace que la situación media de la ciudad no sea excesivamente mala, sino que, por el contrario, un elevado porcentaje de población se encuentre bien servida, aunque por oferta privada.

Cuadro 4. Población en relación con la distancia a los centros de día (total, públicos y privados).

\begin{tabular}{|c|c|c|c|c|c|}
\hline & \multirow{3}{*}{ Población afectada } & \multicolumn{4}{|c|}{ CENTROS DE DÍA } \\
\hline & & \multicolumn{2}{|c|}{ A pie (a más de 20 minutos) } & \multicolumn{2}{|c|}{$\begin{array}{l}\text { Transporte Público (a más de } 40 \\
\text { minutos) }\end{array}$} \\
\hline & & Número & $\begin{array}{c}\text { Porcentaje sobre } \\
\text { el Total de Va- } \\
\text { lencia }\end{array}$ & Número & $\begin{array}{c}\text { Porcentaje sobre } \\
\text { el Total de Va- } \\
\text { lencia }\end{array}$ \\
\hline \multirow{2}{*}{ Total Centros } & Residentes & 149.396 & 19,94 & 3.763 & 0,50 \\
\hline & Mayores de 65 años & 26.411 & 18,02 & 630 & 0,43 \\
\hline \multirow{2}{*}{ Centros públicos } & Residentes & 380.140 & 50,75 & 5.158 & 0,69 \\
\hline & Mayores de 65 años & 69.560 & 47,46 & 890 & 0,61 \\
\hline \multirow{2}{*}{$\begin{array}{l}\text { Centros priva- } \\
\text { dos }\end{array}$} & Residentes & 193.571 & 25,84 & 3.763 & 0,50 \\
\hline & Mayores de 65 años & 33.929 & 23,15 & 630 & 0,43 \\
\hline
\end{tabular}

Fuente: Elaboración propia.

Por la naturaleza del servicio, es evidente que las residencias para la tercera edad no implican un desplazamiento diario del usuario. Sin embargo, exponemos aquí los resultados de accesibilidad por un doble motivo: en primer lugar, para poder compararlos con el resto de servicios considerados en el estudio y conocer su patrón espacial, y en segundo lugar, por enfatizar el aspecto relacionado con la cercanía y el espacio vital, es decir, con el entorno habitual del usuario del servicio. Es cierto que en el caso de las residencias son muchas veces las familias las que deciden, pero dicha decisión puede verse afectada por una estructura de la oferta fundamentalmente privada que dificulta que los familiares puedan seleccionar una residencia en función de su ubicación, sino que son otros aspectos (coste, calidad, variedad en el servicio, cercanía a la residencia de los hijos, etc.) los que determinan la decisión.

Por otra parte, el acceso a una plaza en residencia pública supone el desplazamiento del usuario fuera de la ciudad, donde éstas son más abundantes, con los consecuentes problemas de lejanía, aislamiento y dificultad de acceso por parte de familiares y amigos. Con una estructura de oferta pública equilibrada en el territorio, la localización de residencias y centros de día, es decir, la cercanía al usuario, o a su familia, sería un elemento clave para la decisión y facilitaría que los mayores residentes pudieran seguir vinculados a su entorno, a su barrio (muchas veces también es el barrio en el que reside la familia), y ejercitar el verdadero envejecimiento activo, sin discriminación, siempre contando con que la calidad de la oferta del servicio fuera elevada y de similares características.

A continuación, cabe analizar los mismos resultados, pero únicamente en el caso de las áreas de la ciudad que han sido calificadas como más vulnerables. En dichas áreas, de escala inferior al barrio, el bajo nivel so- 
Cuadro 5. Población en relación con la distancia a las residencias para mayores (total, públicos y privados).

\begin{tabular}{|c|c|c|c|c|c|}
\hline & \multirow{3}{*}{ Población afectada } & \multicolumn{4}{|c|}{ RESIDENCIAS } \\
\hline & & \multicolumn{2}{|c|}{ A pie (a más de 20 minutos) } & \multicolumn{2}{|c|}{$\begin{array}{l}\text { Transporte Público (a más de } 40 \\
\text { minutos) }\end{array}$} \\
\hline & & Número & $\begin{array}{c}\text { Porcentaje sobre } \\
\text { el Total de Va- } \\
\text { lencia }\end{array}$ & Número & $\begin{array}{c}\text { Porcentaje sobre } \\
\text { el Total de Va- } \\
\text { lencia }\end{array}$ \\
\hline \multirow{2}{*}{ Total Centros } & Residentes & 82.751 & 11,05 & 3.816 & 0,51 \\
\hline & Mayores de 65 años & 13.912 & 9,49 & 626 & 0,43 \\
\hline \multirow{2}{*}{ Centros públicos } & Residentes & 612.970 & 81,83 & 5.288 & 0,71 \\
\hline & Mayores de 65 años & 114.780 & 78,32 & 932 & 0,64 \\
\hline \multirow{2}{*}{$\begin{array}{l}\text { Centros priva- } \\
\text { dos }\end{array}$} & Residentes & 82.751 & 11,05 & 5.288 & 0,71 \\
\hline & Mayores de 65 años & 13.912 & 9,49 & 932 & 0,64 \\
\hline
\end{tabular}

Fuente: Elaboración propia.

cio económico coincide con una mala accesibilidad a los servicios destinados a la población mayor. Hemos destacado aquí sólo la accesibilidad a pie, poniendo el mismo umbral que en el análisis anterior: veinte minutos. Se ha cartografiado, pues, la localización del servicio, las áreas con un componente principal 1 negativo (a distintos niveles que indican diferente grado de vulnerabilidad) y la accesibilidad a pie por encima de los veinte minutos señalados. Aquellas más vulnerables (por encima de 2,5 desviaciones estándar) y a más de veinte minutos a pie al servicio más cercano (sea público o privado) se señalan en rojo (mapas 3, 4 y 5). Se ha trabajado con el total, aunque, como se ha señalado, en el caso de los Centros Municipales de Actividades todos son públicos, mientras que, en el caso de las residencias, casi todas son privadas. Se ha decidido así, en esta primera aproximación, porque esta es la estructura general actual y real en la ciudad y porque es posible desarrollar diferentes políticas de apoyo a las personas que superen la dicotomía público-privado, como subvenciones, conciertos, ayudas personales a las situaciones de dependencia, etc. La mejora de la oferta pública es una cuestión de decisión política que supone considerar aspectos como inversiones en equipamientos y en personal a medio y largo plazo.

Como resultado concluyente, cabe señalar que la oferta de servicios a los mayores sigue diferentes modelos de localización según tipología y, por tanto, la población residente en las áreas más vulnerables de la ciudad se ve afectada de diferente forma según el servicio de que se trate.

Los mapas resultantes muestran la localización de las zonas vulnerables o de intervención prioritaria, es decir, la localización de la mayor parte de la anteriormente denominada pobreza senior. En ellas, la población mayor afectada por una lejanía a alguno de los servicios considerados asciende a 191.775 personas, de los cuales, 36.537 son mayores de 65 años.

En el caso de los Centros Municipales de Actividades, 842 residentes en áreas vulnerables se encuentran a más de 20 minutos a pie (cuadro 6). Ello supone menos del $2 \%$ de la población total residente en estas áreas, aunque si consideramos sólo a los mayores de 65 años, el porcentaje asciende ligeramente, hasta el $2,3 \%$, lo que indica que una parte del territorio vulnerable está especialmente envejecido.

Si consideramos los centros de día, tanto públicos como privados, el porcentaje de residentes mayores en áreas vulnerables a más de 20 minutos andando es del 50,37\%. La mitad de los residentes en estas zonas tendrían dificultades para acudir a pie a este tipo de centros, pues les quedan demasiado lejos. Las cifran de acceso a los Centros de Día públicos aún son mayores: el 78,9\% de los mayores de 65 años residentes en las áreas vulnerables se encuentran a más de 20 minutos a pie del Centro de Día público más cercano (cua- 

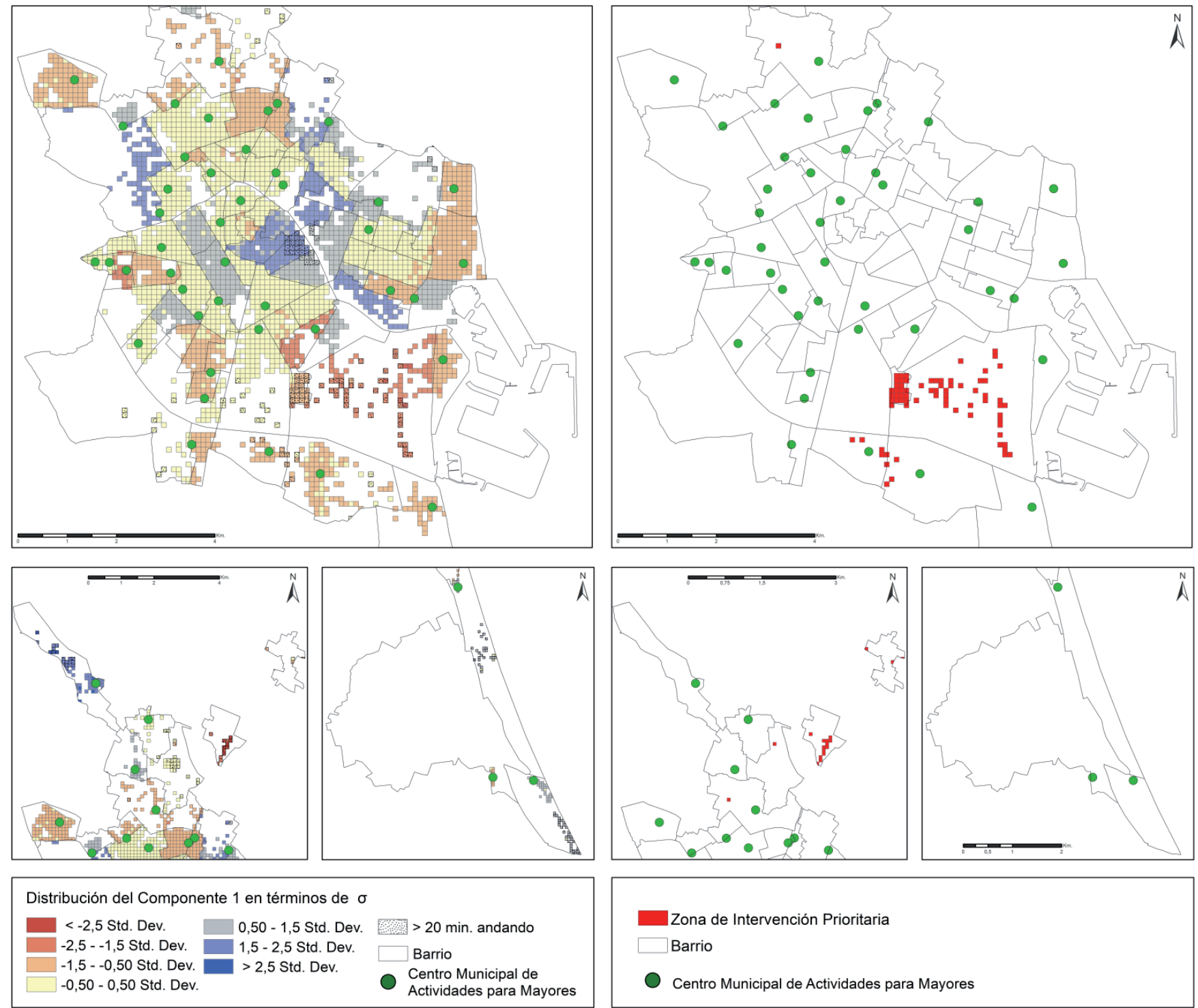

Mapa 3. Nivel socioeconómico (Componente $1 \mathrm{ACP}$ ) y accesibilidad a pie a los Centros Municipales de Actividades para Mayores y zonas de intervención prioritaria en Valencia.

Fuente: Elaboración propia.

dro 6). En el caso de las residencias (total) es del 19,21\%. La diferencia fundamental está en la oferta pública y privada. En este último caso, el de las residencias, si consideramos la distancia en minutos a los centros públicos, las cifras se multiplican: más de 36.000 residentes se ven afectados, lo que supone la práctica totalidad de los mayores de 65 años empadronados en estas zonas vulnerables: el 98,8\% (cuadro 6).

Dado los resultados obtenidos con la aplicación de la metodología propuesta, podemos afirmar que se trata de una herramienta con gran potencial y sencilla de aplicar, siempre que se cuente con la información sociodemográfica detallada a nivel de portal (detalle de escala), tanto de la oferta del servicio como de la demanda. El Padrón Municipal proporciona dicha información, aunque su elaboración puede resultar costosa y generar resistencias en la administración competente dada la necesidad de cumplir con la ley de protección de datos. En el caso de la ciudad de Valencia, los resultados generales son muy positivos, aunque siempre es posible detectar espacios donde las necesidades, reales o potenciales, aún no están completamente cubiertas. Se trata de detectar estos barrios o áreas para calificarlos como prioritarios en cuanto a provisión de servicios o de transporte público específico.

En definitiva, el trabajo aquí presentado versa en la combinación entre la delimitación de las áreas (barrios o parte de los mismos) de menor nivel socioeconómico con las necesidades de cercanía a los servicios 

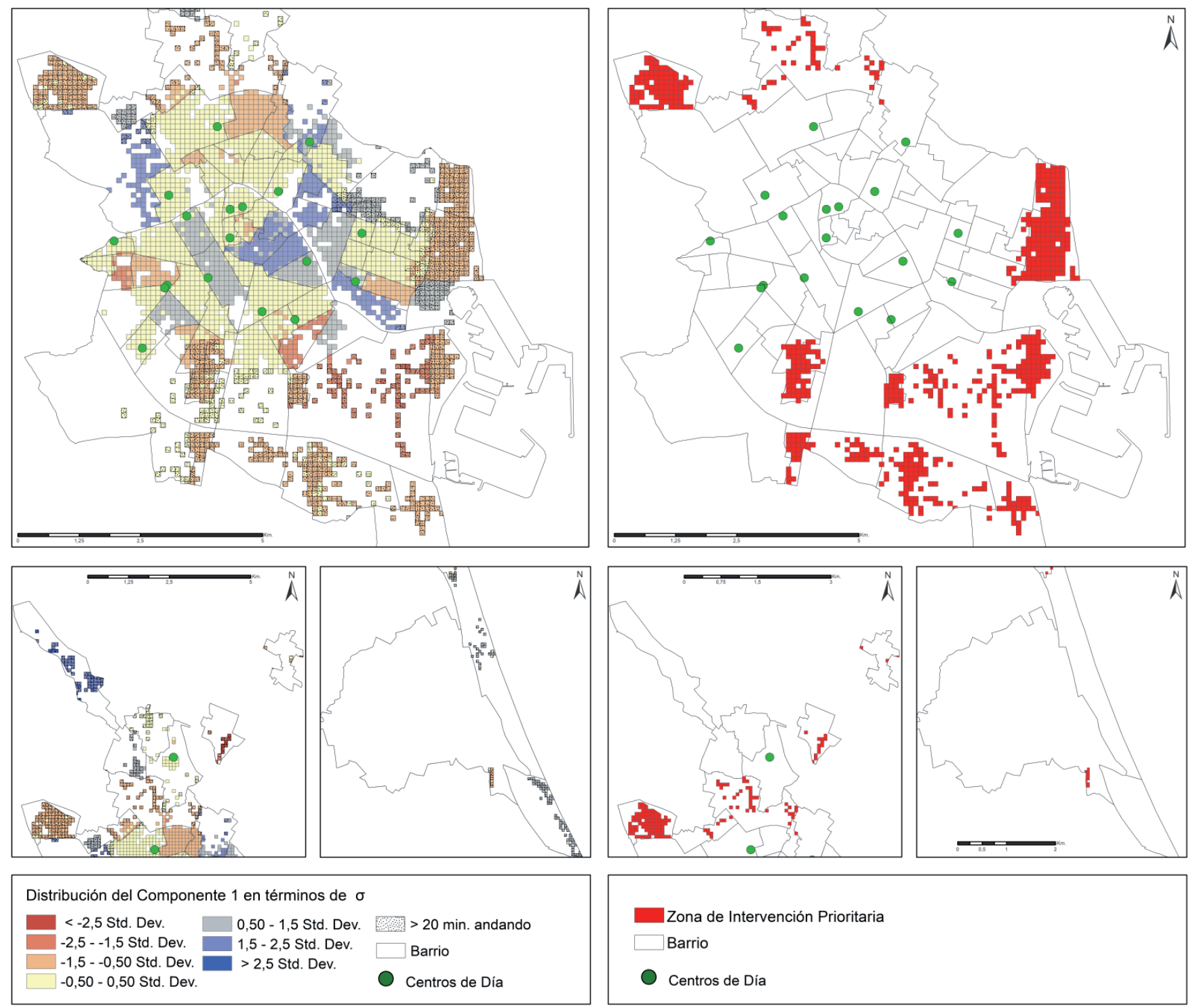

Mapa 4. Nivel socioeconómico (Componente 1 ACP) y accesibilidad a pie a los Centros de Día para Mayores y zonas de intervención prioritaria en Valencia. Fuente: Elaboración propia.

especializados por parte de la población mayor. El estudio de caso no es más que un ejemplo de las posibilidades que esta metodología presenta para poder ser aplicada a otros espacios, ciudades o regiones, en las que se cuente con la información estadística detallada de las personas a nivel de portal. La potencialidad del SIG depende de la calidad (exactitud locacional) de la información, por lo que la metodología propuesta podría modificarse dependiendo de la disponibilidad de datos más precisos.

Por último, cabe señalar que ciertamente existen estudios de accesibilidad a otro tipo de servicios (Calvo et al., 2001, Alfonso et al., 2016, Cazacu et al., 2016, Rode et al., 2017, Pitarch et al., 2018, Pitarch-Garrido, 2018, Zazzi et al. 2018,), la mayor parte centrados en las formas de transporte; sin embargo, son relativamente escasos los artículos científicos centrados en la accesibilidad a los servicios y equipamientos dirigidos a las personas mayores (Nieto-Masot, et al., 2019), a pesar del reto que supone el envejecimiento de la población en las sociedades actuales más avanzadas, en particular en los espacios urbanos. Cabe destacar algunas de las aportaciones recientes puntuales como el análisis del caso de Bangkok (Srichuae et al., 2016), la accesibilidad a los espacios verdes y espacios públicos para la población mayor (Artmann, et al., 2017, Yung et al., 2016) y el estudio de la demanda de los servicios de salud en las ciudades canadienses (Shah, et al., 2016). Todos ellos estudios de interés, sin embargo, no en la línea del que exponemos en el presente artículo. 

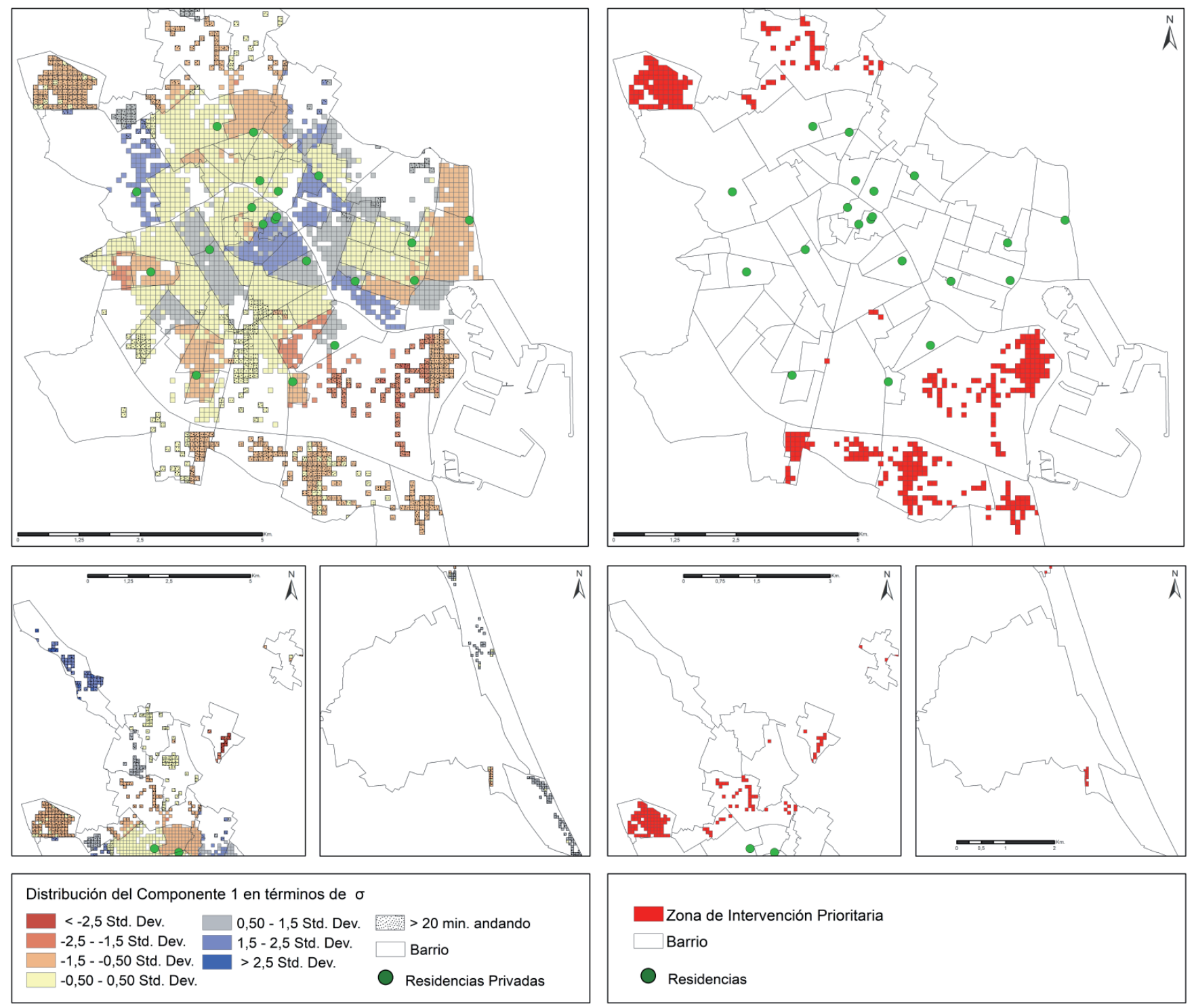

Mapa 5. Nivel socioeconómico (Componente 1 ACP) y accesibilidad a pie a las Residencias y zonas de intervención prioritaria en Valencia. Fuente: Elaboración propia.

Cuadro 6. Población mayor de 65 años residente en las áreas vulnerables de Valencia a más de 20 minutos a pie del centro de servicios más cercano.

\begin{tabular}{|l|l|c|c|}
\hline \multirow{2}{*}{$\begin{array}{l}\text { Centros Municipales de } \\
\text { Actividades }\end{array}$} & \multicolumn{1}{|c|}{ Titularidad } & Mayores de 65 años & $\begin{array}{c}\text { Porcentaje sobre el Total de Población ma- } \\
\text { yor de 65 años en Zonas Vulnerables }\end{array}$ \\
\cline { 2 - 4 } & Total & 842 & 2,30 \\
\hline \multirow{2}{*}{ Centros De Día } & Total & 18.403 & 50,37 \\
\cline { 2 - 4 } & Públicos & 28.809 & 78,85 \\
\hline & Privados & 19.326 & 52,89 \\
\hline \multirow{3}{*}{ Residencias } & Total & 7.017 & 19,21 \\
\cline { 2 - 4 } & Públicos & 36.118 & 98,85 \\
\cline { 2 - 4 } & Privados & 7.017 & 19,21 \\
\hline
\end{tabular}

Fuente: Elaboración propia a partir de datos del Padrón Municipal. 


\section{CONCLUSIONES}

La investigación presentada permite aplicar una metodología de análisis espacial para contribuir al conocimiento de una realidad, la accesibilidad de las personas mayores en situación económica vulnerable, a los servicios a ellas destinados, con el mayor detalle que permiten las estadísticas y la tecnología SIG. Se trata de un análisis que no por ser un clásico de la Geografía, deja de tener interés en la actualidad. La percepción de los tomadores de decisiones no es suficiente para responder de la manera más adecuada a las necesidades sociales, pues precisan de herramientas y metodologías que, con la información disponible a nivel local, corroboren o no dichas percepciones.

En Valencia son escasas las áreas o barrios que necesitan una intervención integral con el fin de acercar la oferta de servicios a los mayores, sin embargo, un análisis en detalle permite detectar los servicios que son menos accesibles por parte de la población en general, pero de la población más directamente afectada en particular. La búsqueda de la equidad espacial y social, ambas vinculadas estrechamente, en un espacio urbano dinámico y densamente poblado, ha de considerar a toda la población, sea cual sea su nivel de renta y también la localización de su residencia, pues todos deben disfrutar de una buena accesibilidad a los servicios. La aparición de nuevas formas de vulnerabilidad, en este caso, asociadas a la llamada pobreza senior, suponen un reto para las políticas públicas y de planificación que hay que acometer antes de que se conviertan en problema.

Es preciso, en este punto, tener en consideración dos cuestiones: por una parte, la diferencia entre la oferta pública y la privada, y por otra, la necesaria proximidad a los servicios, la vinculación con el barrio. La oferta privada no responde a patrones relacionados con la equidad, sino con el mercado, y, aun así, en la ciudad de Valencia, algunos servicios privados para la tercera edad, como son las residencias, están localizadas de manera que se reparten el mercado potencial de manera bastante equilibrada. En este caso, el principal problema de la oferta pública es su escasez. Sin embargo, la oferta pública de servicios debería garantizar plazas a todos los posibles usuarios, sin importar sus características personales. La segunda cuestión tiene que ver con el entorno habitual de las personas, que no deben verse obligadas a abandonar su barrio en especial a una edad en la que la cotidianeidad es importante para anclarse en el presente y mantener una vida larga y de calidad, es decir, un envejecimiento activo. En este sentido, la acción pública en forma de oferta de servicios de calidad para las personas mayores, en su entorno del barrio, en particular si estas son dependientes, es capital.

En conclusión, y aunque es evidente que toda la población mayor, sea cual sea su nivel de renta, debe disfrutar de una buena accesibilidad (cercanía) a los servicios públicos de proximidad, la realidad es que, en una situación de recesión económica y contención del gasto, es necesario priorizar las inversiones y orientarlas allí donde éstas son más urgentes o necesarias, en particular cuando estamos hablando de un segmento de la población con necesidades muy específicas y crecientemente afectado por nuevas formas de pobreza y exclusión, como la que hemos venido denominando aquí pobreza senior. El análisis realizado ayuda, en este sentido, a la toma de decisiones públicas, favorece la movilidad sostenible (a pie) y genera bienestar en la población. En el caso analizado, la ciudad de Valencia, se ha desarrollado una metodología que permite avanzar en estudios de geografía aplicada y que puede transferirse a otros entornos urbanos. Los umbrales de tiempo pueden variar dependiendo de las necesidades de la investigación, y la demanda puede proyectarse para trabajar sobre escenarios a más largo plazo. La detección de las áreas en riesgo de exclusión facilita herramientas para el diseño de políticas de planificación urbana que luchen contra la exclusión, tanto social como espacial, en pro de una mejora de la calidad de vida de toda la ciudadanía.

\section{REFERENCIAS}

Abellán García, A. y Pujol Rodríguez, R. (2016). Un perfil de las personas mayores en España, 2016. Indicadores estadísticos básicos. Madrid, Informes Envejecimiento en red, n. 14. Recuperado de http://envejecimiento.csic.es/documentos/ documentos/enred-indicadoresbasicos16.pdf 
Ajuntament de València (2018). Població major de 64 anys a la ciutat de València. Valencia: Oficina de Estadística del Ajuntament de València. Recuperado de: file://F:/PUBLICAR/TERCERA\%20EDAD\%20para\%20REA/Majors64_2018.pdf

Alfonso, J., Duquesne, C., Sánchez, N., Menéndez, J. M., Blanco, N. \& Melero, T. (2016). Urban mobility data managementthe OPTICITIES project and the Madrid standardization proposal. Transportation Research Procedia, 14, 1260-1269. doi: https://doi.org/10.1016/j.trpro.2016.05.198

Amin, A, (2002). Ethnicity and the multicultural city: living with diversity. Environment and Planning A, 34, 959-980. doi: https://doi.org/10.1068/a3537

Andreotti, A., Mingione, E. \& Polizzi, E. (2012). Local welfare systems: a challenge for social cohesion. Urban studies, 49 (9), 1925-1940. doi: https://doi.org/10.1177/0042098012444884

Artmann, M., Chen, X., Iojă, C., Hof, A., Onose, D., Poniży, L., ... \& Breuste, J. (2017). The role of urban green spaces in care facilities for elderly people across European cities. Urban Forestry \& Urban Greening, 27, 203-213. doi: https://doi. org/10.1016/j.ufug.2017.08.007

Berjano, E. y Pinazo, S. (2003). ¿Están preparadas las grandes ciudades para atender a los ancianos dependientes? Revista multidisciplinar de gerontología, 13 (4), 252-266.

Blockland, T. et al. (eds.) (2016). Creating the unequal city. The exclusion consequences of everyday routines in Berlin. Nueva York: Routledge. doi: https://doi.org/10.4324/9781315574639

Calvo, J.L. et al. (2001). Análisis, diagnóstico y ordenación de equipamientos mediante formulaciones cartografiables: valoración de la accesibilidad y requerimientos de la asistencia hospitalaria en la CC.AA. de la Rioja mediante la técnica de potenciales. Berceo, 141, 247-268.

Campelo, P., Izaola, A., Urrutia, V. y Zubero, (2014). Vulnerabilidades sociodemográficas en las ciudades, en I. Subirats, J. y Martí.Costa, M. (eds) Ciudades, vulnerabilidades y crisis en España. Centro de Estudios Andaluces, Consejería de Presidencia, Junta de Andalucía, 109-145.

Cazacu, V. A., Ionescu, B. G., y Tudora, D. (2016). Analyze of the population accesibility to the essential services of general interest in the historical region of moldavia. International Multidisciplinary Scientific GeoConference: SGEM: Surveying Geology \& mining Ecology Management, 3, 51-56. doi: https://doi.org/10.5593/SGEM2016/B23/S11.007

Doyle, Y., McKee, M., Rechel, B. \& Grundy, E. (2009). Meeting the challenge of population ageing. BMJ (Clinical research ed). Recuperado de http://researchonline.Ishtm.ac.uk/4658/1/Meeting\%20the\%20challenge\%20of\%20population\%20ageing_1_\%20The\%20BMJ.pdf

Falkingham, J.C., Héran, F. \& Vaupel, J.W. (2011). Europe's citizens should have a choice. Toward a new policy of life-course flexibility. Population Policy Compact. Recuperado de file:///C:/Users/usuario/Downloads/population_policy_compact_01-2011.pdf

Garrocho, C. y Campos J. (2006). Un indicador de accesibilidad a unidades de servicios clave para ciudades mexicanas: fundamentos, diseño y aplicación, Economía, Sociedad y Territorio. VI (22), 1-60.

Iglesias Souto, M.I., Real Deus, J.E., Dosil Maceira, A., Mayo Pais, M.E. y Taboada Ares, E.M. (2018). Asignación de servicios sociales a personas mayores: revisión y modelo de toma de decisiones. Cuadernos de Trabajo Social, 31 (2), $417-430$. doi: https://doi.org/10.5209/CUTS.55454

López-Roldán, P., Fachelli, S. (2016). Análisis factorial. En P. López-Roldán y S. Fachelli, Metodología de la Investigación Social Cuantitativa. Bellaterra (Cerdanyola del Vallès): Dipòsit Digital de Documents, Universitat Autònoma de Barcelona. $1^{\text {a }}$ edición, versión 3. Recuperado de http://ddd.uab.cat/record/142928

Marquet, S. y Miralles, C. (2014). La proximidad en Barcelona. Un análisis desde los tiempos de desplazamiento cotidianos. Ciudades, $17,99-120$

McCann, P. (2017). Urban futures, population ageing and demographic decline. Cambridge Journal of Regions, Economics and Society, 10, 543-557. doi: https://doi.org/10.1093/cjres/rsx009

Nieto-Masot, A., Engelmo-Moriche, A. y Cárdena-Alonso, G. (2019). La Distribución Territorial de Recursos Sanitarios y Socio-Sanitarios Públicos para Población Mayor en Extremadura. Revista de Estudios Andaluces, 37, 141-160. doi: https://doi.org/10.12795/rea.2019.i37.07

OCDE (2009). Policies for Healthy Ageing: An Overview. OECD Health Working Papers, $\mathrm{n}^{\circ} 42,32$. Recuperado de https:// www.oecd-ilibrary.org/docserver/226757488706.pdf?expires=1556653672\&id=id\&accname=guest\&checksum=2624BF4EF969EE29FC676809247F32B8 
OCDE (2012). Compact cities policies: A comparative Assessment. Paris: OCDE. Recuperado de http://www.oecd.org/ greengrowth/compact-city-policies-9789264167865-en.htm Último acceso: 21 de diciembre de 2018.

OCDE (2015a). Ageing in cities. Paris: OCDE. Recuperado de http://www.oecd.org/regional/ageing-in-cities9789264231160-en.htm Último acceso: 21 de diciembre de 2018.

OCDE (2015b). Sustainable Urban Development Policies in Ageing Societies. Paris: OCDE. Recuperado de http://www. oecd.org/governance/regional-policy/sustainable-urban-development-policies-in-ageing-societies.htm Último acceso: 21 de diciembre de 2018.

OMS (2001). Campaña de la OMS por un envejecimiento activo. OMS. Recuperado de https://www.who.int/ageing/publications/alc_elmanual.pdf?ua=1 Último acceso: 20 de diciembre de 2018.

OMS (2012). La buena salud añade vida a los años. Información general para el día mundial de la salud 2012. OMS. Recuperado de http://apps.who.int/iris/bitstream/handle/10665/75254/WHO_DCO_WHD_2012.2_spa.pdf;jsessionid=BOF995F38336DD389AE16C686DA0126B?sequence=1 Último acceso: 20 de diciembre de 2018.

OMS (2015). Informe mundial sobre el envejecimiento y la salud. OMS. Recuperado de http://apps.who.int/iris/bitstream/handle/10665/186466/9789240694873_spa.pdf?sequence=1 Último acceso: 20 de diciembre de 2018.

Pitarch-Garrido, M.D., Salom, J. y Fajardo, F. (2018). Detección de barrios vulnerables a partir de la accesibilidad a los servicios públicos de proximidad. El caso de la ciudad de Valencia. Anales de Geografía de la Universidad Complutense, 38 (1), 61-85. doi: https://doi.org/10.5209/AGUC.60469

Pitarch-Garrido, M.D. (2018). Social Sustainability in Metropolitan Areas: accessibility and Equity in the Case of the Metropolitan Area of Valencia (Spain). Sustainability, 10, 371. doi: https://doi.org/10.3390/su10020371

Roberts, K. \& Chapman, T. (2017). Elderly people as active users of health and social care. Londres: Routledge.

Rode, P., Floater, G., Thomopoulos, N., Docherty, J., Schwinger, P., Mahendra, A., \& Fang, W. (2017). Accessibility in cities: transport and urban form. In Disrupting mobility (pp. 239-273). Springer, Cham. doi: https://doi.org/10.1007/978-3319-51602-8_15

Shah, T. I., Bell, S., \& Wilson, K. (2016). Spatial accessibility to health care services: identifying under-serviced Neighbourhoods in Canadian urban areas. PloS one, 11(12). Recuperado de https://journals.plos.org/plosone/article?id=10.1371/journal.pone.0168208. https://doi.org/10.1371/journal.pone.0168208

Sancho Comíns, J. y Reinoso Moreno, D. (2012). La delimitación del ámbito rural: una cuestión clave en los programas de desarrollo rural. Estudios Geográficos, Vol. LXXIII, 273, 599-624. doi: https://doi.org/10.3989/estgeogr.201221

Silva, T., Caravau, H. \& Campelo, D. (2017). Information needs about public and social services of Portuguese elderly. En 3rd International Conference on Information and Communication Technologies for Ageing Well and e-Health. doi: 10.5220/0006284900460057.

Srichuae, S., Nitivattananon, V., \& Perera, R. (2016). Aging society in Bangkok and the factors affecting mobility of elderly in urban public spaces and transportation facilities. Iatss Research, 40 (1), 26-34. doi: https://doi.org/10.1016/j. iatssr.2015.12.004

Yanguas, J.J., Cilveti, A., Hernández, S., Pinazo, S., Roig, S. y Segura, C. (2018). El reto de la soledad en la vejez. Zerbitzuan: Gizarte zerbitzuetarako aldizkaria. Revista de servicios sociales, 66, 61-75. doi: https://doi.org/10.5569/11347147.66 .05

Yung, E. H., Conejos, S., \& Chan, E. H. (2016). Social needs of the elderly and active aging in public open spaces in urban renewal. Cities, 52, 114-122. doi: https://doi.org/10.1016/j.cities.2015.11.022

Zazzi, M., Ventura, P., Caselli, B. y Carra, M. (2018). GIS-based monitoring and evaluation system as an urban planning tool to enhance the quality of pedestrian mobility in Parma. In Town and Infrastructure Planning for Safety and Urban Quality: Proceedings of the XXIII International Conference on Living and Walking in Cities (LWC 2017). June 15-16, 2017. Brescia, Italy: CRC Press (p. 87). doi: https://doi.org/10.1201/9781351173360-14 